\title{
A Randomized Trial Comparing Short versus Prolonged Hemostasis with Rescue Recanalization by Ipsilateral Ulnar Artery Compression: Impact on Radial Artery Occlusion-The RESCUE- RAO Trial
}

\author{
Dmitrii V. Ognerubov $\mathbb{D}^{1},{ }^{1}$ Alexander Sedaghat $\mathbb{D}^{2}{ }^{2}$ Sergey I. Provatorov, \\ Andrey S. Tereshchenko $\mathbb{D}^{1},{ }^{1}$ Olivier F. Bertrand, ${ }^{3}$ Ivo Bernat $\mathbb{D}^{4},{ }^{4}$ Goar K. Arutyunyan $\mathbb{D},{ }^{1}$ \\ Olga A. Pogorelova $\mathbb{D D}^{1}{ }^{1}$ Maria I. Tripoten ${ }^{1}{ }^{1}{ }^{1}$ Tatyana V Balakhonova. $\left(\mathbb{D},{ }^{1}\right.$ \\ Anatoliy N. Samko $\mathbb{D}^{1}{ }^{1}$ and Evgeny V. Merkulov ${ }^{1}{ }^{1}$ \\ ${ }^{1}$ National Medical Research Center of Cardiology, Ministry of Health of Russia, Moscow, Russia \\ ${ }^{2}$ Medizinische Klinik und Poliklinik II, Universitätsklinikum Bonn, Bonn, Germany \\ ${ }^{3}$ Quebec Heart-Lung Institute, Quebec, Canada \\ ${ }^{4}$ University Hospital and Faculty of Medicine Pilsen, Pilsen, Czech Republic
}

Correspondence should be addressed to Dmitrii V. Ognerubov; dr.ognerubov@gmail.com

Received 18 March 2020; Revised 26 August 2020; Accepted 5 October 2020; Published 23 October 2020

Academic Editor: Salvatore De Rosa

Copyright ( 12020 Dmitrii V. Ognerubov et al. This is an open access article distributed under the Creative Commons Attribution License, which permits unrestricted use, distribution, and reproduction in any medium, provided the original work is properly cited.

\begin{abstract}
Background. Despite the enormous benefits of radial access, this route is associated with a risk of radial artery occlusion (RAO). Objective. We compared the incidence of RAO in patients undergoing transradial coronary angiography and intervention after short versus prolonged hemostasis protocol. Also we assessed the efficacy of rescue 1-hour ipsilateral ulnar artery compression if RAO was observed after hemostasis. Material and Methods. Patients referred for elective transradial coronary procedures were eligible. After $6 \mathrm{~F}$ radial sheath removal, patients were randomized to short (3 hours) $(n=495)$ or prolonged ( 8 hours) ( $n=503)$ hemostasis and a simple bandage was placed over the puncture site. After hemostasis was completed, oximetry plethysmography was used to assess the patency of the radial artery. Results. One thousand patients were randomized. Baseline characteristics were similar between both groups with average age $61.4 \pm 9.4$ years $(71 \%$ male $)$ and PCI performed on half of the patients. The RAO rate immediately after hemostasis was $3.2 \%$ in the short hemostasis group and $10.1 \%$ in the prolonged group $(p<0.001)$. Rescue recanalization was successful only in the short group in $56.2 \%(11 / 19)$; at hospital discharge, RAO rates were $1.4 \%$ in the short group and $10.1 \%$ in the prolonged group $(p<0.001)$. Conclusion. Shorter hemostasis was associated with significantly less RAO compared to prolonged hemostasis. Rescue radial artery recanalization was effective in $>50 \%$, but only in the short hemostasis group.
\end{abstract}

\section{Introduction}

Radial access (RA) has become the gold standard for coronary angiography (CAG) and percutaneous coronary interventions (PCI) within the last decade. The use of this access is associated with reduced rates of access site complications, MACE, and improved clinical outcomes, including survival [1-3]. In addition to clinical advantages, RA is linked to socioeconomic benefits including reduced inhospital stays and costs [2]. The main convenience of RA results from the superficial location of the radial artery, its smaller diameter, and the fact that the radial artery can be easily compressed while allowing patient mobility [4]. A relevant downside of RA is the relatively high incidence of 
radial artery occlusion (RAO). This mostly asymptomatic complication can be found in $1 \%-30 \%$ of patients after radial access [5-9]. Although clinical symptoms are uncommon and critical ischemia is rarely encountered [10], the presence of RAO makes this artery unsuitable for repeated access or other clinical uses including arterial grafting in coronary bypass surgery or in the context of arteriovenous fistula surgery for dialysis. Prevention, early detection, and effective treatment of RAO are therefore of clinical importance in maintaining patency of this access route. Adequate compression should be applied to reduce local bleeding complications and also to achieve adequate hemostasis. The compressive pressure should be reduced earlier, but with careful monitoring for local bleeding complications or rebleeding $[11,12]$. In this study, we aimed to compare the incidence of RAO in patients undergoing both diagnostic CAG and PCI using 2 different regimens of radial artery hemostasis. We further compared the efficacy of ipsilateral ulnar artery compression for the treatment of early RAO in both groups.

\section{Material and Methods}

2.1. Study Cohort and Study Design. The RESCUE-RAO Trial (a randomized trial comparing short versus prolonged hemostasis with rescue recanalization by ipsilateral ulnar artery compression-impact on radial artery occlusion) was performed at a large cardiology center in the Russian Federation. Patients referred for diagnostic CAG or PCI using RA were enrolled. The study flow diagram is seen in Figure 1.

Patients had to be older than 18 years old and needed to provide written informed consent to participate in this study. Due to more aggressive preclinical treatment and less predictable anticoagulation, patients with acute coronary syndrome as well as patients with documented coagulopathy or under treatment with oral anticoagulants were excluded. The study protocol and design were approved by the local institutional review committee and ethics committee of the National Medical Research Center of Cardiology in Moscow. All patients signed informed consent to participate in the study before the inclusion. Patients were pretreated prior to catheterization with acetylsalicylic acid (125 mg daily) and clopidogrel ( $75 \mathrm{mg}$ daily). Anatomy of both radial arteries was evaluated by ultrasound before the procedures in all patients. In the short group, pressure dressings were applied for a mean of 3 hours, according to the previous unpublished pilot study, whereas in the prolonged group, pressure dressings were applied for 8 hours according to the local clinical experience [13].

The primary endpoint of this study was defined as the presence of 24-hour RAO after the procedure confirmed by oximetry-plethysmography test and duplex ultrasound. Secondary endpoints included (a) rate of successful recanalization of initial RAO and (b) rate of local bleeding and hematoma.

RAO was defined as negative oximetry/plethysmography test [14] (absence of plethysmographic signals from pulse oximeter placed on the index during ulnar compression) immediately after the end of hemostasis, that is, when the pressure dressing was taken off. Duplex sonography was performed in patients with suspected RAO by the oximetry/ plethysmography test. All patients who had suspected RAO were examined with duplex sonography 24 hours after the procedure. Local upper extremity hematoma was categorized according to the EASY scale [15]. Bleeding from the access site was defined as the bleeding that occurred 3 hours after the intervention and which required a reexamination by a physician and the replacement of the dressing.

2.2. Procedural Specifics. Preprocedural duplex ultrasound was performed in all patients. Vessel patency, tortuosity, and the internal diameters were examined with a standard ultrasound device (Phillips IU-22, Philips N.V., Netherlands) using a linear probe L3-9. The images were obtained by dedicated vascular ultrasonographists, who were blinded to the study.

Radial artery catheterization was performed according to the Seldinger technique as previously described $[12,16]$. Local anesthesia was performed by a subcutaneous injection of $2 \%$ lidocaine at the puncture site. After insertion of a transradial access sheath (Radifocus Introducer II, Terumo, Europe N.V.), 5,000 IU of unfractionated heparin (UFH) was injected for diagnostic coronary angiography, whereas $100 \mathrm{IU} / \mathrm{kg}$ UFH was used for PCI patients. Procedures were performed via $6 \mathrm{Fr}$ catheters. A spasmolytic cocktail was not routinely given in this study. Additional nitroglycerin was given by the discretion of the treating physician. In this study, radial artery hemostasis was achieved through compression of the radial artery using a pressure dressing with a roller placed directly in the puncture site $[7,8]$.

\subsection{Detection and Treatment of the Radial Artery Occlusion} (RAO). After removal of the compression bandage, patients were evaluated for RAO with oximetry/plethysmographic test $[16,17]$. The pulse oximetry curve at the index finger was recorded with a standard pulse oximeter (Armed YX302, Russia). A positive result was defined as the detection of oscillations on the oximetry curve during compression of the ipsilateral ulnar artery $[18,19]$. A negative result, that is, suspected RAO, was defined as the absence of the oximetry curve [19]. After initial testing, a second oximetry/plethysmography test was repeated 24 hours after the procedure in all patients. In patients with $\mathrm{RAO}$, additional ultrasound of the forearm arteries was performed. When RAO was detected after initial hemostasis, we performed 1-hour compression of the ipsilateral ulnar artery. At the point of best pulsation $2-3 \mathrm{~cm}$ above the wrist, a TR band (Terumo, Japan) was inflated with $15-18 \mathrm{~cm}^{3}$ of air. Complete occlusion of the ulnar artery was confirmed by the absence of the curve on a pulse oximeter located on the index finger. Excess air was removed so as not to hinder venous outflow and not to create additional pressure on the occluded radial artery and surrounding wrist tissues. One hour later, the ulnar artery was released and the oximetry/plethysmography test was repeated. Successful recanalization was noted 


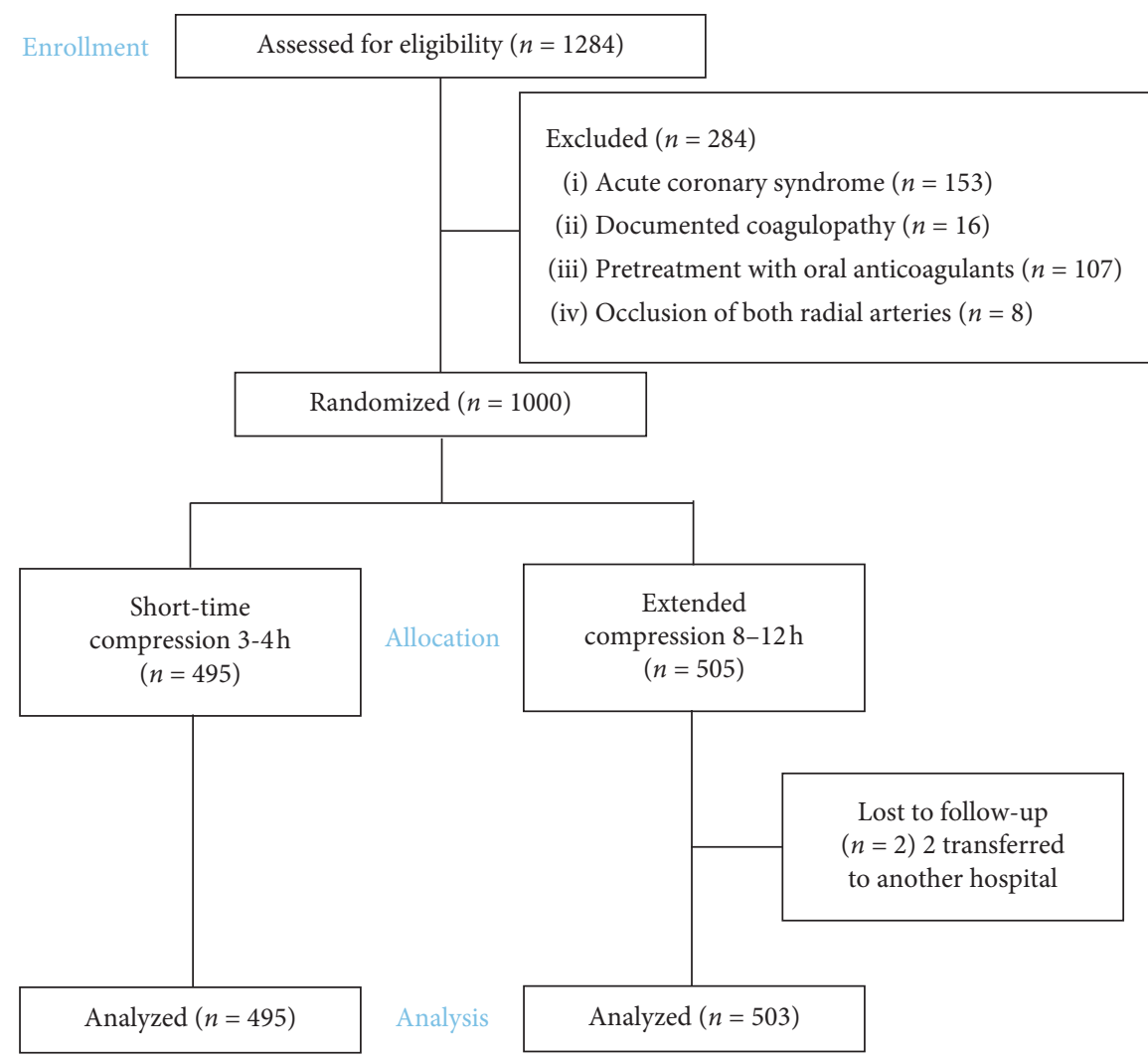

Figure 1: Study flow diagram.

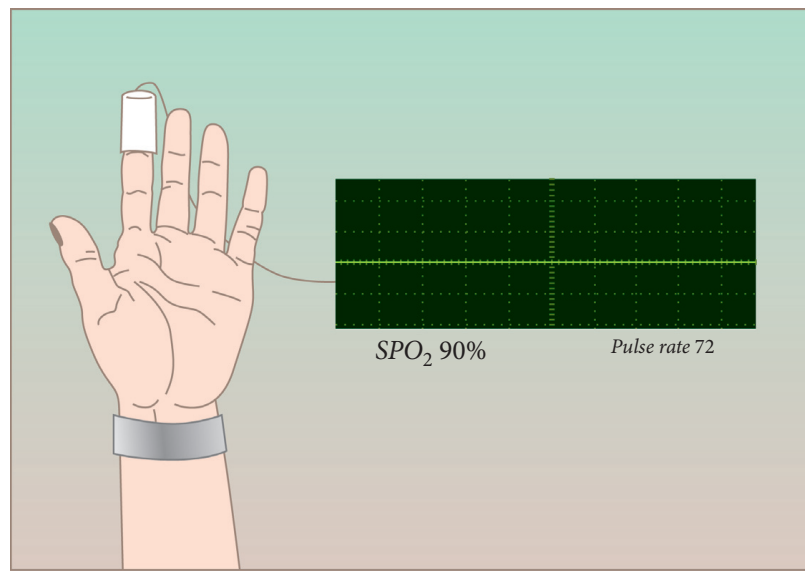

(a)

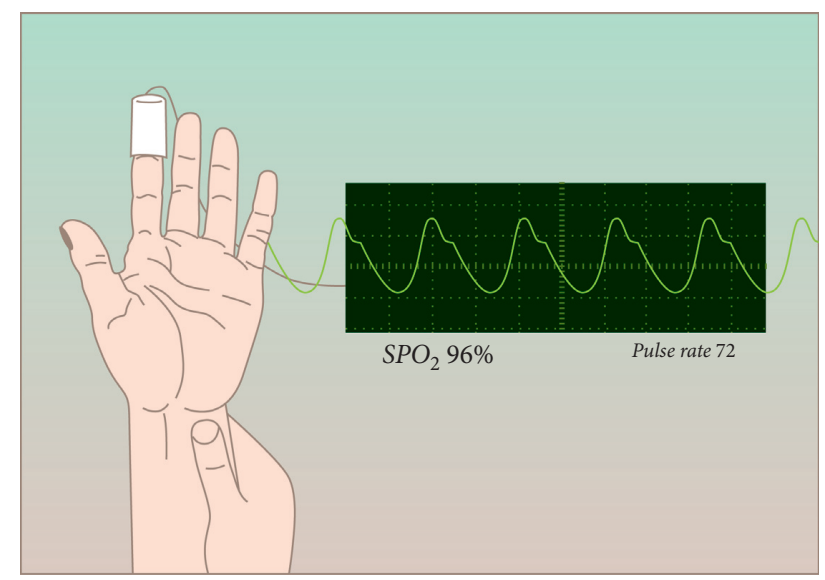

(b)

FIgURE 2: Scheme of radial artery recanalization. (a) Compression of the ulnar artery with TR band for one hour (no plethysmographic waveform (RAO)). (b) Repeating pulse oximeter test and recording the appearance of plethysmographic waveform indicating that recanalization of RAO was successful.

upon return of the signal on the oximetry/plethysmography test. The scheme of the recanalization technique is presented in Figures 2 and 3.

2.4. Statistical Analysis. For statistical processing of the results, we used the statistical program $R$ 3.4.4 software ( $\mathrm{R}$ Core Team [2014], Vienna, Austria). For all patient quality characteristics, the relative frequency of all possible parameter values was indicated. When comparing different groups, the following statistical criteria were used: (1) Student's $t$-test for independent samples was used for quantitative characteristics distributed according to normal law; (2) for quantitative characteristics that have an abnormal distribution, the Mann-Whitney test was used; (3) differences among categorical variables were 


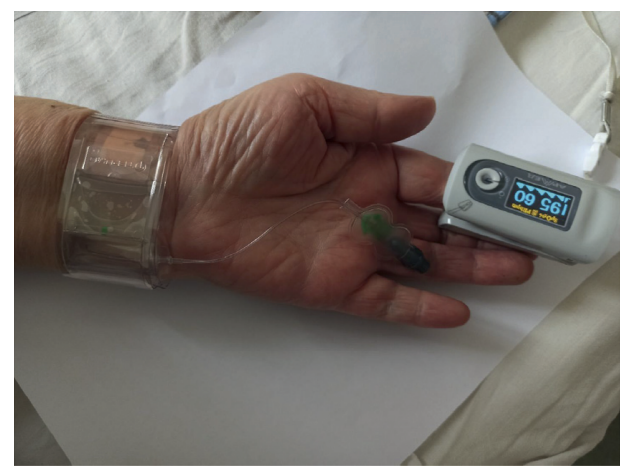

FIGURE 3: Compression of the ulnar artery with TR band with radial flow restoration (the appearance of plethysmographic waveform).

evaluated using the chi-square test or Fisher exact test. Differences were considered statistically significant at $p<0.05$.

The sample size calculation was performed using a superiority design with the assumed incidence of the primary endpoint of RAO of $8,8 \%$ in prolonged hemostasis [8] and $2.8 \%$ (reference data derived from local registry) in short-time hemostasis. Overall, 494 patients per group (total $=988$ ) were deemed adequate to achieve $90 \%$ power considering an alpha error of 0.01 , assuming a $5 \%$ rate of crossover from short-time hemostasis to prolonged hemostasis due to bleeding or hematoma [20].

\section{Results}

From 2015 to 2018, from 1284 all-comers patients screened, a total of 1000 patients undergoing diagnostic CAG or PCI were randomized with sealed envelopes in two groups. Patients in both groups were randomized before sheath removal to receive either short $(n=495)$ or prolonged hemostasis $(n=505)$. Two patients from the extended group were transferred to another hospital and could not be evaluated.

As for clinical and procedural characteristics, no significant differences were observed between the two groups (Tables 1 and 2). Overall, patients were $61.4 \pm 9.4$ years of age and the majority were male (70.9\%). Among concomitant diseases, arterial hypertension was found in $90.4 \%$ of cases, whereas dyslipidemia and diabetes mellitus were present in $28.3 \%$ and $22.5 \%$, respectively. Smokers accounted for $25.3 \%$ of all patients.

The RAO rate immediately after hemostasis was $3.2 \%$ in the short hemostasis group and $10.1 \%$ in the prolonged group $(p<0.001)$. Rescue recanalization was successful only in the short group $11 / 19(56.2 \%)$ versus none in the prolonged group $(p<0.001)$. The primary endpoint of 24 -hour RAO occurred in $5.8 \%$ (58/998) of patients. The incidence of RAO was significantly lower in patients undergoing shorttime hemostasis $(1.4 \%$ versus $10.2 \%, p<0.001)$ (Table 3 ).

The frequency of bleeding from the access site in the groups with short and prolonged hemostasis was similar $(0.8 \%$ and $0.8 \%$, resp., $p=1.0)$. In addition, no differences in the incidence of minor hematoma (EASY grade 1-2) were seen $(2.2 \%$ versus $2.2 \%, p=0.79)$. More pronounced access site hematoma (EASY grade 3 ) occurred only in 2 cases with prolonged hemostasis $(0.4 \%)$ and in one case of the short group $(0.2 \%)$. None of the patients developed major access site hematoma (EASY grade 4-5) and needed surgical therapy or transfusion.

\section{Discussion}

The results of our randomized study demonstrate a statistically, significant, and clinically relevant reduction in the incidence of RAO detected $24 \mathrm{~h}$ after the procedure using short-time compression after radial access for both CAG and PCI. Rescue recanalization via ipsilateral compression of the ulnar artery was effective only in the short-time compression group. At the same time, there were no differences regarding bleeding or the development of hematoma at the access site between the 2 regimes.

With the ascent of radial artery access, prevention, and management of radial artery, occlusion has become a relevant issue $[7,12,17]$. Overall, our results regarding RAO are compared well with other studies published on this topic. The incidence of RAO varies greatly from 1.1 to $33 \%$ $[7,9,17,21]$. Whereas Uhlemann et al. reported a relatively high incidence of RAO with $33.1 \%$ using $6 \mathrm{~F}$ introducers [9], RAO occurred in $5.8 \%$ of our patients. In contrast to our analysis, the authors used a commercially available dosage compression device. In the presented analysis, hemostasis devices, such as the Terumo TR Band, were not routinely used. Although the application of the air compression device has been found to be associated with a lower incidence of RAO [16], the additional cost of these devices is a relevant limitation regarding their use, especially in developing countries. In addition, the protocol, which has to be applied, has several limitations, including the need for larger involvement of the postprocedural care team and repeated evaluation of radial flow. Given the use of a simple pressure dressing, our results indicate the safety and feasibility of this approach with acceptable rates of RAO as well as bleeding [8].

Additionally, recanalization via ipsilateral compression of the ulnar artery led to a successful recanalization in $56.2 \%$, only in those patients undergoing short-term compression. Otherwise, recanalization could not be achieved in any patient undergoing traditional hemostasis, while in the group of short hemostasis, $56.2 \%$ with RAO in short hemostasis were recanalized $(p<0.001)$. It remains possible that even more prolonged use $(>1 \mathrm{~h})$ of ipsilateral ulnar artery compression could have a better result on the RAO recanalization rate. Bernat et al. showed comparable success of recanalization in $71 \%$ (5 out of 7) in a group of 5000 IU of heparin and $31 \%$ (4 out of 13) in 2000 IU heparin group, but they evaluated only patients after CAG [22]. In contrast, Tian et al. recanalized $98.9 \%$ of RAO, which is likely to be linked to prolonged manual compression of the ulnar artery in their study, which was performed for $4.1 \pm 1.2 \mathrm{~h}$ [23]. In clinical routine, there are several potential reasons for the failure of recanalization of the radial artery. Unsuccessful recanalization may be caused by incorrect compression or 
TABLE 1: Baseline characteristics.

\begin{tabular}{lccc}
\hline & Prolonged hemostasis $(n=503)$ & Short-time hemostasis $(n=495)$ & $p$ \\
\hline Age & $61.6 \pm 9.9$ & $61.1 \pm 9.0$ & 0.43 \\
Height & $172.9 \pm 8.0$ & $172.9 \pm 6.6$ & 0.77 \\
Weight & $84.1 \pm 11.06$ & $83.9 \pm 13.6$ & 0.85 \\
Body mass index & $28.1 \pm 3.8$ & $28.1 \pm 2.9$ & 0.96 \\
Smoking & $131(26 \%)$ & $122(24 \%)$ & 0.69 \\
Diabetes mellitus & $106(21 \%)$ & $121(24 \%)$ & 0.27 \\
Dyslipidemia & $134(27 \%)$ & $149(30 \%)$ & 0.33 \\
Male & $367(73 \%)$ & $341(69 \%)$ & 0.21 \\
Arterial hypertension & $448(89 \%)$ & $456(92 \%)$ & 0.12 \\
Dose of heparin, 1000 IU & $7.42 \pm 2.92$ & $7.53 \pm 2.73$ & 0.42 \\
Diameter of radial artery & $2.32 \pm 0.38$ & $2.3 \pm 0.5$ & 0.58 \\
Body surface area & $2 \pm 0.19$ & $2 \pm 0.16$ & \\
PCI & $252(50 \%)$ & $248(50 \%)$ &
\end{tabular}

TABle 2: Periprocedural characteristics.

\begin{tabular}{lccc}
\hline & Prolonged hemostasis $(n=503)$ & Short-time hemostasis $(n=495)$ & $p$ \\
\hline Crossover & $4(0.8 \%)$ & $6(1.2 \%)$ & 0.73 \\
Fluoroscopy time, min & $10.2 \pm 10$ & $10.1 \pm 9.3$ & 0.98 \\
Procedure time & $23.8 \pm 10.8$ & $23.7 \pm 10.1$ & 0.84 \\
Radial artery spasm & $30(6 \%)$ & $31(6.2 \%)$ & 0.93 \\
Artery/sheath ratio & $0.89 \pm 0.1$ & $0.89 \pm 0.1$ & 0.76 \\
\hline
\end{tabular}

TABle 3: Frequency of complications.

\begin{tabular}{|c|c|c|c|}
\hline & Prolonged hemostasis $(n=503)$ & Short-time hemostasis $(n=495)$ & $p$ \\
\hline Rate of RAO after removal & $51(10.1 \%)$ & $16(3.2 \%)$ & $<0.001$ \\
\hline Rate of 24-hour RAO & $51(10.1 \%)$ & $7(1.4 \%)$ & $<0.001$ \\
\hline Bleeding & $4(0.8 \%)$ & $4(0.8 \%)$ & 1.0 \\
\hline Hematoma (stage 1-2) & $11(2.2 \%)$ & $11(2.2 \%)$ & 1,0 \\
\hline Hematoma ( $\geq$ stage 3 ) & $1(0.2 \%)$ & $2(0.4 \%)$ & 1.0 \\
\hline
\end{tabular}

insufficient compression force. Also, too short periods of compression may be causative in this context [24]. According to the work of Tian et al., compression can be performed up to 6 hours with virtually no subjective symptoms [23]. In our work, the clinical manifestations of RAO included the absence of pulsation in a third of the cases and the sensation of paresthesia and numbness of the thumb and index finger. During the ipsilateral compression, the symptoms of ischemia of the hand were not found in any patient. At the same time, Nagai et al. reported that $14 \%$ of patients complained of pain in the hand, but this is due to the fact that the researchers used this technique for more than 3 hours [23].

From a pathophysiological perspective, the cause of acute postcatheterization occlusion is occlusive thrombosis [25]. Thrombosis occurs at the site of injury of the vascular wall. In the area of local injury of the vascular wall and slow blood flow during hemostasis, a soft red blood clot easily forms. At this stage, spontaneous restoration of the radial artery patency is possible. Later after retraction and compaction of the red thrombus, the probability of its recanalization goes down. Heparin contributes to the spontaneous recanalization of soft fresh blood clots, its effect just about 4 hours after the procedure [26]. So, rapid restoration of blood flow could be in the first hours after the development of occlusion. Early removal of the compression bandage independently increases the likelihood of restoration of blood flow in the radial artery. In the same line of argument, it will be proper to measure that "prophylactic" ipsilateral ulnar compression may have the highest efficacy in "preventing" $\mathrm{RAO}$, as proven by randomized and nonrandomized data $[18,27]$.

Ultrasound is the gold standard for the detection of the RAO and the most accurate method, which allows evaluating the flow rate, its direction (antegrade or retrograde), and the arteries' anatomy. Flow absence along with information about anatomy (curves, loops, etc.) is very important for future interventions [27-29]. However, the oximetry/plethysmography test is a simple and inexpensive bedside method. This method is highly sensitive and can be equally useful in assessing of the radial artery patency $[6,7,16,18,29]$. We found that rates of RAO by oximetry/ plethysmography and duplex ultrasound were equal.

The incidence of bleeding $(0.8 \%)$ and local hematoma $(2.2 \%)$ in both groups was comparable. The reduction of the duration of hemostasis did not lead to an increase in 
bleeding complications and hematomas, compared with the other studies $[4,6,9,16,18,21]$.

Radial artery hemostasis is an important issue in the transradial approach since the rate of radial artery occlusion is not negligible in the clinical practice. However, it should be clearly stated that dedicated hemostatic devices, although expensive, associated with patent hemostasis technique are the gold standard in order to prevent RAO [29].

The safety and simplicity of the ipsilateral ulnar artery compression, with the lack of need for complex equipment, combined with its highly significant efficacy and safety decrease the incidence of RAO. This fact could encourage most interventional cardiologists to embrace the technique as default protocol, in addition to other established best practices to reduce RAO after transradial access.

\section{Limitations}

Our study did not include patients with acute coronary syndrome; this does not allow extending the conclusions of this work to this cohort of patients. Our investigation is a singlecenter study with a limited number of patients. Further research is needed to investigate methods of recanalization of acute occlusion of the radial artery. Early RAO was diagnosed by the oximetry/plethysmography test; an ultrasound examination of these patients was performed only on the following day.

\section{Conclusion}

Shorter hemostasis was associated with significantly less RAO compared to prolonged hemostasis. Rescue radial artery recanalization by one-hour ipsilateral ulnar artery compression was effective only in the short hemostasis group. It remains possible that even more prolonged use (> $1 \mathrm{~h}$ ) of ipsilateral ulnar artery compression could have a better result on the RAO recanalization rate.

\section{Abbreviations}

RA: Radial access

PCI: Percutaneous coronary interventions

CAG: Coronary angiography

RAO: Radial artery occlusion

UFH: Unfractionated heparin.

\section{Data Availability}

The data used to support the findings of this study are available from the corresponding author upon request.

\section{Conflicts of Interest}

The authors declare that there are no conflicts of interest regarding the publication of this paper.

\section{References}

[1] A. J. Chase, E. B. Fretz, W. P. Warburton et al., "Association of the arterial access site at angioplasty with transfusion and mortality: the M.O.R.T.A.L study (Mortality benefit of
Reduced Transfusion after percutaneous coronary intervention via the Arm or Leg)," Heart, vol. 94, no. 8, pp. 1019-1025, 2008.

[2] A. D. Kugelmass, D. J. Cohen, P. P. Brown, A. W. Simon, E. R. Becker, and S. D. Culler, "Hospital resources consumed in treating complications associated with percutaneous coronary interventions," The American Journal of Cardiology, vol. 97, no. 3, pp. 322-327, 2006.

[3] G. Ferrante, S. V. Rao, P. Jüni et al., "Radial versus femoral access for coronary interventions across the entire spectrum of patients with coronary artery disease," JACC: Cardiovascular Interventions, vol. 9, no. 14, pp. 1419-1434, 2016.

[4] S. Rathore, R. H. Stables, M. Pauriah et al., "A randomized comparison of TR band and radistop hemostatic compression devices after transradial coronary intervention," Catheterization and Cardiovascular Interventions, vol. 76, no. 5, pp. 660-667, 2010.

[5] S. Plante, W. J. Cantor, L. Goldman et al., "Comparison of bivalirudin versus heparin on radial artery occlusion after transradial catheterization," Catheterization and Cardiovascular Interventions, vol. 76, no. 5, pp. 654-658, 2010.

[6] G. Hahalis, K. Aznaouridis, G. Tsigkas et al., "Radial artery and ulnar artery occlusions following coronary procedures and the impact of anticoagulation: ARTEMIS (radial and ulnar ARTE ry occlusion Meta-analys IS ) systematic review and meta-analysis," Journal of the American Heart Association, vol. 6, no. 8, Article ID e005430, 2017.

[7] O. F. Bertrand, S. V. Rao, S. Pancholy et al., "Transradial approach for coronary angiography and interventions," JACC: Cardiovascular Interventions, vol. 3, no. 10, pp. 10221031, 2010.

[8] D. V. Ognerubov, S. I. Provatorov, A. S. Tereshchenko et al., "Rate of complications at early removal of compression bandage after transradial coronary angiography," Kardiologiia, vol. 59, no. 1, pp. 79-83, 2019.

[9] M. Uhlemann, S. Gielen, A. Linke et al., "The Leipzig prospective vascular ultrasound registry in radial artery catheterization,” European Heart Journal, vol. 32, p. 955, 2012.

[10] H. Chim, K. Bakri, and S. L. Moran, "Complications related to radial artery occlusion, radial artery harvest, and arterial lines," Hand Clinics, vol. 31, no. 1, pp. 93-100, 2015.

[11] A. Ç. Aykan, T. Gökdeniz, I. Gül et al., "Comparison of low dose versus standard dose heparin for radial approach in elective coronary angiography?" International Journal of Cardiology, vol. 187, pp. 389-392, 2015.

[12] V. Dangoisse, A. Guédès, P. Chenu et al., "Usefulness of a gentle and short hemostasis using the transradial band device after transradial access for percutaneous coronary angiography and interventions to reduce the radial artery occlusion rate (from the Prospective and Randomized CRASOC I, II, and III Studies)," The American Journal of Cardiology, vol. 120, no. 3, pp. 374-379, 2017.

[13] G. R. Barbeau, F. Arsenault, L. Dugas, S. Simard, and M. M. Larivière, "Evaluation of the ulnopalmar arterial arches with pulse oximetry and plethysmography: comparison with the Allen's test in 1010 patients," American Heart Journal, vol. 147, no. 3, pp. 489-493, 2004.

[14] O. F. Bertrand, "Acute forearm muscle swelling post transradial catheterization and compartment syndrome: prevention is better than treatment!" Catheterization and Cardiovascular Interventions, vol. 75, no. 3, pp. 366-368, 2010.

[15] S. Pancholy, J. Coppola, T. Patel, and M. Roke-Thomas, "Prevention of radial artery occlusion-patent hemostasis 
evaluation trial (PROPHET study): a randomized comparison of traditional versus patency documented hemostasis after transradial catheterization," Catheterization and Cardiovascular Interventions, vol. 72, no. 3, pp. 335340, 2008.

[16] M. Rashid, C. S. Kwok, S Pancholy et al., "Radial artery occlusion after transradial interventions: a systematic review and meta-Analysis," Journal of the American Heart Association, vol. 5, no. 1, Article ID e002686, 2016.

[17] S. B. Pancholy, I. Bernat, O. F. Bertrand, and T. M. Patel, "Prevention of radial artery occlusion after transradial catheterization," JACC: Cardiovascular Interventions, vol. 9, no. 19, pp. 1992-1999, 2016.

[18] S. Dharma, S. Kedev, T. Patel, S. V. Rao, and I. C. Gilchrist, "Different spasmolytic regimens (nitroglycerin vs verapamil) and the incidence of radial artery occlusion after transradial catheterization," Journal of Invasive Cardiology, vol. 30, no. 12, pp. 461-464, 2018 Dec.

[19] S. J. Pocock, Clinical Trials: A Practical Approach, Wiley, Hoboken, NJ, USA, 1983.

[20] A. Aminian, S. Saito, A. Takahashi et al., "Impact of sheath size and hemostasis time on radial artery patency after transradial coronary angiography and intervention in Japanese and nonJapanese patients: a substudy from RAP and BEAT (Radial Artery Patency and Bleeding, Efficacy, Adverse lini) randomized multicenter trial," Catheterization and Cardiovascular Interventions, vol. 92, pp. 1-8, 2018.

[21] X.-L. Bi, X.-H. Fu, X.-S. Gu et al., "Influence of puncture site on radial artery occlusion after transradial coronary intervention," Chinese Medical Journal, vol. 129, no. 8, pp. 898-902, 2016.

[22] J. Tian, Y.-S. Chu, J. Sun, and T.-M. Jiang, "Ulnar artery compression," Chinese Medical Journal, vol. 128, no. 6, pp. 795-798, 2015.

[23] S. Nagai, S. Abe, T. Sato et al., "Ultrasonic assessment of vascular complications in coronary angiography and angioplasty after transradial approach," The American Journal of Cardiology, vol. 83, no. 2, pp. 180-186, 1999.

[24] A. R. Zankl, M. Andrassy, C. Volz et al., "Radial artery thrombosis following transradial coronary angiography: incidence and rationale for treatment of symptomatic patients with low-molecular-weight heparins," Clinical Research in Cardiology, vol. 99, no. 12, pp. 841-847, 2010.

[25] J. Hirsh and R. Raschke, "Heparin and low-molecular-weight heparin," Chest, vol. 126, no. 3, pp. 188S-203S, 2004.

[26] M. J. Koutouzis, C. D. Maniotis, G. Avdikos, A. Tsoumeleas, C. Andreou, and Z. S. Kyriakides, "ULnar artery Transient compression facilitating Radial Artery patent hemostasis (ULTRA): a novel technique to reduce radial artery occlusion after transradial coronary catheterization," Journal of Invasive Cardiology, vol. 28, pp. 451-454, 2016.

[27] I. Bernat, O. F. Bertrand, R. Rokyta et al., "Efficacy and safety of transient ulnar artery compression to recanalize acute radial artery occlusion after transradial catheterization," The American Journal of Cardiology, vol. 107, no. 11, pp. 16981701, 2011.

[28] S. B. Pancholy, "Transradial access in an occluded radial artery: new technique," Journal of Invasive Cardiology, vol. 19, pp. 541-544, 2007.

[29] I. Bernat and A. Aminian, S. Pancholy et al., "Best practices for the prevention of radial artery occlusion after transradial diagnostic angiography and intervention," JACC: Cardiovascular Interventions, vol. 12, no. 22, pp. 2235-2246, 2019. 